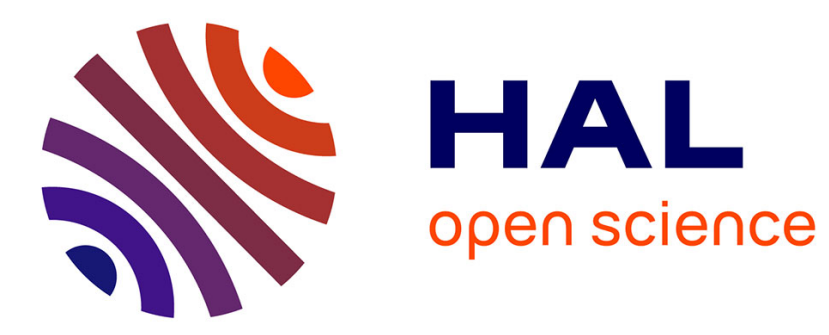

\title{
Multitemporal SAR Image Decomposition into Strong Scatterers, Background, and Speckle
}

\author{
Sylvain Lobry, Loïc Denis, Florence Tupin
}

\section{To cite this version:}

Sylvain Lobry, Loïc Denis, Florence Tupin. Multitemporal SAR Image Decomposition into Strong Scatterers, Background, and Speckle. IEEE Journal of Selected Topics in Applied Earth Observations and Remote Sensing, 2016, 9 (8), pp.3419 - 3429. 10.1109/JSTARS.2016.2555579 . ujm-01376896

\section{HAL Id: ujm-01376896}

\section{https://hal-ujm.archives-ouvertes.fr/ujm-01376896}

Submitted on 5 Oct 2016

HAL is a multi-disciplinary open access archive for the deposit and dissemination of scientific research documents, whether they are published or not. The documents may come from teaching and research institutions in France or abroad, or from public or private research centers.
L'archive ouverte pluridisciplinaire HAL, est destinée au dépôt et à la diffusion de documents scientifiques de niveau recherche, publiés ou non, émanant des établissements d'enseignement et de recherche français ou étrangers, des laboratoires publics ou privés. 


\title{
Multi-temporal SAR image decomposition into strong scatterers, background, and speckle
}

\author{
Sylvain Lobry, Loïc Denis, Florence Tupin
}

\begin{abstract}
Speckle phenomenon in synthetic aperture radar (SAR) images makes their visual and automatic interpretation a difficult task. To reduce strong fluctuations due to speckle, total variation (TV) regularization has been proposed by several authors to smooth out noise without blurring edges. A specificity of SAR images is the presence of strong scatterers having a radiometry several orders of magnitude larger than their surrounding region. These scatterers, especially present in urban areas, limit the effectiveness of TV regularization as they break the assumption of an image made of regions of constant radiometry. To overcome this limitation, we propose in this paper an image decomposition approach.

There exists numerous methods to decompose an image into several components, notably to separate textural and geometrical information. These decomposition models are generally recast as energy minimization problems involving a different penalty term for each of the components. In this framework, we propose an energy suitable for the decomposition of SAR images into speckle, a smooth background and strong scatterers, and discuss its minimization using max-flow/min-cut algorithms. We make the connection between the minimization problem considered, involving the LO pseudo-norm, and the generalized likelihood ratio test used in detection theory. The proposed decomposition jointly performs the detection of strong scatterers and the estimation of the background radiometry.

Given the increasing availability of time series of SAR images, we consider the decomposition of a whole time series. New change detection methods can be based on the temporal analysis of the components obtained from our decomposition.
\end{abstract}

Index Terms-SAR, Image decomposition, TV, LO, Change detection.

\section{INTRODUCTION}

Due to the coherent nature of SAR imaging technique, SAR images suffer from strong fluctuations related to the speckle phenomenon. While containing information about the sub-resolution texture of the scene, speckle is often regarded as undesirable noise for image interpretation tasks. Speckle is then typically modeled as a multiplicative noise. Another peculiarity of SAR images is their high dynamic range: manmade structures such as buildings, fences or transmission towers produce very strong back-scattering, with intensities much larger than the surrounding area. Such scatterers are especially numerous in urban areas. A quick overview of

S. Lobry is with LTCI, CNRS, Télécom ParisTech, Université Paris-Saclay, 75013, Paris, France and the Centre National d'Études Spatiales (CNES), Toulouse, France, e-mail: sylvain.lobry@telecom-paristech.fr.

L. Denis is with the Laboratoire Hubert Curien, UMR 5516 CNRS, Université de Saint-Etienne and Télécom Saint-Etienne, Saint-Etienne, France, e-mail: loic.denis@univ-st-etienne.fr.

F. Tupin is with LTCI, CNRS, Télécom ParisTech, Université Paris-Saclay, 75013, Paris, France, e-mail: florence.tupin@telecom-paristech.fr

Manuscript received FIXME; revised FIXME. speckle reduction reveals that most methods do not directly account for the presence of those strong scatterers.

The simplest way to reduce speckle fluctuations is spatial multi-looking which amounts to averaging pixel values within a sliding window. This speckle variance reduction is obtained at the cost of a resolution loss proportional to the size of the averaging window. While it may produce satisfying results in homogeneous areas, it strongly blurs textured areas, edges between regions and bright scatterers.

Numerous approaches have been proposed to prevent the introduction of blur by mixing values from distinct regions. Lee et al. [1] locally select the best window among a few oriented windows. The IDAN algorithm [2] builds an adaptive window by region growing. Several methods have been derived from the non-local means (NL-means) approach by Buades $e t$ al. [3]. These methods select similar pixels in an extended window based on patch-similarity [4]-[10]. Another family of methods reduce speckle by regularization, i.e., by computing the maximum a posteriori estimate under a given prior. Wavelet-based approaches model the distribution of wavelet coefficients [11]-[13]. Total variation (TV) regularization penalizes variations between neighboring pixels while preserving sharp edges [14]. Total variation has been applied to the regularization of SAR amplitudes [15]-[17], SAR intensities [18], [19] and log-transformed intensities [20] using different optimization strategies (discrete optimization by graph-cuts, gradient descent, Douglas-Rachford splitting or the alternating directions method of multipliers). We refer the reader to the two recent review papers [21] and [22] for an in-depth analysis of speckle reduction methods.

Isolated strong scatterers are challenging for speckle reduction methods because they generally break the statistical assumptions made about the radar scene: repetition of similar patches within the search window (patch-based methods), sparse representation in the wavelets domain (wavelets-based methods), or piece-wise constant regions (TV minimization). It is then necessary to identify and process these points separately to prevent from spreading these large values. Rather than building a statistical model of spatial configurations (edges, textures) in SAR images, many works directly model heterogeneity due both to the presence of strong scatterers and to geometrical features under a stationary assumption [23], [24]. It is then possible to derive estimators robust to fluctuations of radiometry within the neighborhood [25].

On the one hand, statistical models that describe geometrical structures such as edges in SAR images generally fail to account for point-like strong scatterers; on the other hand, models of heterogeneous clutter based on a stationary assump- 
tion are lacking geometrical information. In this paper, we attempt to account for both geometrical content and strong scatterers in an explicit way, i.e., we recover the geometrical part and detect the strong scatterers.

Strong scatterers can be detected using likelihood ratio tests [26], [27]. These detectors compare the values in the center of a window with the rest of the window, considered as purely background. In dense urban areas, the presence of other point-like scatterers in the vicinity strongly deteriorates the performance.

It is thus necessary to perform jointly the detection of strong scatterers with the estimation of the background radiometry. The decomposition method that we propose in the present paper achieves such a joint detection of point-like scatterers and estimation of the background. It applies to multi-temporal stacks of images by detecting scatterers at each date, even if they are not present at other dates due to some change occurring.

The idea of decomposing the SAR signal into several components is not new and led to very successful approaches in different SAR modalities. The reconstruction of a sparse distribution of scatterers has first been introduced in [28], where a smoothing and a sparsity inducing penalty were applied to the same image. Sparse regularization has been further investigated under the concept of compressed sensing [29], [30] and successfully applied to tomographic SAR focusing [31]. Beyond the recovery of point-like sources, decomposition under a dictionary of SAR signatures has been considered in [32]. Detection of permanent scatterers and possibly of distributed scatterers is at the heart of permanent scatterers processing in multi-pass interferometry [33], [34]. Decompositions into different scattering behavior is also central to the analysis of polarimetric images, see for example [35]. Other decomposition schemes have been considered, in particular to analyze the anisotropic behavior of scatterers from subaperture decomposition.

The approach followed in this paper is in the spirit of image decomposition methods studied in the field of image processing [36]-[39]. These works consider the decomposition of a given image into several components, one typically containing geometrical information (i.e., edges), another capturing the textural information (oscillating components), and the remaining component with the noise. These decompositions are obtained by enforcing the sum of all components to match the initial image while each component gets penalized by a different regularization term promoting a different property (piece-wise constant image, oscillating image). This paper extends the decomposition models presented in [40], [41] in the following ways: we derive and motivate the decomposition model from a connection between scatterers detection and maximum a posteriori estimation with a regularization term based on L0 pseudo-norm (section II). The proposed decomposition model can provide a multi-temporal image of detected scatterers and a smoothly varying multi-temporal background image, or a simpler decomposition into multitemporal scatterers image and a time-invariant background. Computing the decomposition requires solving a challenging large-scale combinatorial problem. We describe two algorith- mic approaches (section III): an exact discrete optimization based on finding the minimum cut in a large 4-D graph [42], and a faster but approximate method based on block processing with overlaps. This fast algorithm is essential for the applicability of the method to large regions and/or temporal stacks. We show on numerical experiments that the choice of the L0 non-convex penalty significantly improves the detection of scatterers compared to the usual L1 norm surrogate $^{1}$ (section IV). Finally, we illustrate how the detected scatterers can be used in a subsequent processing step for change detection purposes (section V-B).

\section{SPARSE + SMOOTH DECOMPOSITION MODEL FOR MULTI-TEMPORAL DATA}

\section{A. From detection to estimation under LO prior}

Let us consider first the problem of detecting strong scatterers in a speckled pixel $i$ of given radiometry $u_{B}$. At each pixel, the detection problem amounts to deciding between two hypotheses: the absence $\left(\mathcal{H}_{0}\right)$ or presence $\left(\mathcal{H}_{1}\right)$ of a strong scatterer in addition to the other scatterers that form the background:

$$
\begin{cases}\mathcal{H}_{0}: & u_{S i}=0 \\ \mathcal{H}_{1}: & u_{S i}>0\end{cases}
$$

where $u_{S i}$ is the radiometry of the additional scatterer.

Under the assumption of uncorrelated speckle and point-like scatterers, this hypothesis test can be performed independently at each pixel by studying the likelihood radio:

$$
\log \frac{\mathrm{p}\left(v_{i} \mid u_{B i}+u_{S i}\right)}{\mathrm{p}\left(v_{i} \mid u_{B i}\right)} \underset{\mathcal{H}_{0}}{\stackrel{\mathcal{H}_{1}}{\gtrless}} \lambda,
$$

with $v_{i}$ the observed amplitude at pixel $i$ (i.e., specklecorrupted) and $\lambda$ a threshold. This likelihood ratio test can not be readily applied since the radiometry $u_{S i}$ is unknown. Replacing $u_{S i}$ by the maximum likelihood estimate leads to the generalized likelihood ratio test (GLRT) [43]:

$$
\max _{u_{S i} \geq 0} \log \mathrm{p}\left(v_{i} \mid u_{B i}+u_{S i}\right) \underset{\mathcal{H}_{0}}{\stackrel{\mathcal{H}_{1}}{\gtrless}} \lambda+\log \left(\mathrm{p}\left(v_{i} \mid u_{B i}\right)\right) .
$$

In words, the presence of an additional scatterer is detected at pixel $i$ if the log-likelihood of the observed amplitude $v_{i}$ is improved at least by $\lambda$ when hypothesizing that a scatterer is present in addition to the background scatterers. The threshold $\lambda$ sets the false alarm rate: increasing $\lambda$ reduces the probability of wrongly selecting hypothesis $\mathcal{H}_{1}$ under $\mathcal{H}_{0}$, at the cost of reducing the detection probability.

This detection problem can be stated as an estimation problem, i.e., as the estimation of the radiometry of the additional scatterer $u_{S i}$, where a zero estimated radiometry corresponds to the absence of the additional scatterer at pixel $i$ :

$$
\widehat{u_{S i}}=\underset{u_{S i} \geq 0}{\arg \min }\left[-\log \mathrm{p}\left(v_{i} \mid u_{B i}+u_{S i}\right)+\lambda \cdot\left(u_{S i} \neq 0\right)\right],
$$

\footnotetext{
${ }^{1}$ note that in SAR compressed sensing [28]-[31] an L1 norm is generally considered because an inversion procedure is involved (focusing); our image decomposition task is easier since it does not require such inversion
} 
where the notation $\lambda \cdot\left(u_{S} \neq 0\right)$ refers to value $\lambda$ whenever $u_{S} \neq 0$, and 0 when $u_{S}=0$. Equation (4) is completely equivalent to equation (3), and can be extended to a whole image. The estimation of the vector of all radiometries $\mathbf{u}_{\mathbf{S}}$, for a given vector of corresponding background radiometries $\mathbf{u}_{\mathbf{B}}$ and under our pixel-independence assumption, is given by:

$$
\widehat{\mathbf{u}_{\mathbf{S}}}=\underset{\mathbf{u}_{\mathbf{S}} \geq 0}{\arg \min }\left[-\sum_{i} \log \mathrm{p}\left(v_{i} \mid u_{B i}+u_{S i}\right)+\lambda\left\|\mathbf{u}_{\mathbf{S}}\right\|_{0}\right],
$$

where the positivity constraint $\mathbf{u}_{\mathbf{S}} \geq 0$ applies componentwise and the L0 pseudo-norm corresponds to the number of non-zero components of vector $\mathbf{u}_{\mathbf{S}}$.

Under Goodman's fully developed speckle model [44], the observed amplitude $v_{t, i}$ at pixel $i$ and time $t$ follows a Rayleigh distribution:

$$
\mathrm{p}\left(v_{t, i} \mid u_{t, i}\right)=\frac{2 v_{t, i}}{u_{t, i}^{2}} \exp \left(\frac{-v_{t, i}^{2}}{u_{t, i}^{2}}\right),
$$

where $u_{t, i}=u_{B t, i}+u_{S t, i}$ is the radiometry at pixel $i$ and time $t$. Other statistical distributions may be considered, for example Rice distribution which is more accurate when one strong scatterer is dominant in the resolution cell $\left(u_{S t, i} \gg u_{B t, i}\right)$. With the choice of Rayleigh distribution, the generalized likelihood ratio test (3) can be obtained in closed form (see Appendix):

$$
\frac{v_{i}^{2}}{u_{B_{i}^{2}}^{2}}-\log \frac{v_{i}^{2}}{u_{B i}^{2}} \underset{\mathcal{H}_{0}}{\stackrel{\mathcal{H}_{1}}{\gtrless}} \lambda+1
$$

We therefore consider Rayleigh distribution in the following. Since the test depends only on the ratio $v_{i} / u_{B i}$ which is Rayleigh distributed with parameter 1 under $\mathcal{H}_{0}$, the threshold $\lambda$ sets a constant false alarm rate (i.e., $\lambda$ needs not be tuned with the level $u_{B}$ ).

\section{B. Joint estimation of the background and of strong scatterers}

Direct applicability of the estimation formulation given in equation (5) is limited because it requires the knowledge of the background radiometry $\mathbf{u}_{\mathrm{B}}$. Estimation of this background radiometry requires to exclude strong scatterers, i.e., the detection of all scatterers in $\mathbf{u}_{\mathbf{S}}$. The two components $\mathbf{u}_{\mathbf{B}}$ and $\mathbf{u}_{\mathbf{S}}$ must thus be jointly estimated:

$$
\begin{aligned}
\left(\widehat{\mathbf{u}_{\mathbf{S}}}, \widehat{\mathbf{u}_{\mathbf{B}}}\right)=\underset{\mathbf{u}_{\mathbf{S}} \geq 0, \mathbf{u}_{\mathbf{B}} \geq 0}{\arg \min }\left[-\sum_{i}\right. & \log \mathbf{p}\left(v_{i} \mid u_{B i}+u_{S i}\right) \\
& \left.+\lambda\left\|\mathbf{u}_{\mathbf{S}}\right\|_{0}+\psi\left(\mathbf{u}_{\mathbf{B}}\right)\right],
\end{aligned}
$$

where $\psi$ is a smoothness term necessary to enforce the regularity of the background and thus prevent problem degeneracy ${ }^{2}$. Regularization term $\psi$ introduces a coupling between all unknowns, i.e., the minimization problem in (8) is not separable. With the L0 term, the optimization problem is combinatorial (non-convex and discontinuous). Given the large scale of the problem (typically millions to billions of unknown pixel radiometries to estimate), two approaches can be considered:

\footnotetext{
${ }^{2}$ in the absence of the smoothness term $\psi$, the decomposition is trivial: $\widehat{\mathbf{u}_{\mathbf{S}}}=\mathbf{0}$ and $\widehat{\mathbf{u}_{\mathbf{B}}}=\widehat{\mathbf{u}_{\mathbf{B}}}{ }^{(\mathrm{ML})} \equiv \arg \min _{\mathbf{u}_{\mathbf{B}} \geq 0}-\sum_{i} \log \mathrm{p}\left(v_{i} \mid u_{B i}\right)$ is the maximum likelihood estimate of the background.
}

- convex relaxation: the L0 term is generally replaced by the L1 norm to turn the combinatorial minimization problem into a convex minimization problem;

- combinatorial optimization: direct minimization of (8) is possible for some specific choices of the regularization term $\psi$.

The first approach suffers from two drawbacks: (i) under speckle noise, the neg-log-likelihood is non-convex, which prevents direct application of standard convex optimization methods; (ii) with the L1 norm, the estimation problem is no longer equivalent to the detection problem. We show in section IV that this leads to a non-constant false alarm rate: scatterers are mainly detected in darker areas.

The second approach consists of applying a combinatorial optimization method to the problem, for a given discretization of the radiometries. It restates the original optimization problem as a minimum cut / maximum flow search on a graph [42]. It applies to discrete optimization problems involving (possibly non-convex) separable terms and convex pairwise terms, and is well suited (i.e., the graph is sparsest) for anisotropic total variation:

$$
T V(\mathbf{u})=\sum_{i \sim j} w_{i, j}\left|u_{i}-u_{j}\right|
$$

where $i \sim j$ indicates that $i$ and $j$ are indices of two neighboring pixels, and $w_{i, j}$ are weights. In the simplest form, only the 4 nearest neighbors are considered and all weights $w_{i, j}$ are equal to 1 . To reduce the metrication effects, farther neighbors can also be considered, with smaller weights, see for example [45]. In the following, we will consider a smoothness term $\psi$ based on total variation (9) so that a graph-cut method can be applied to solve the non-convex minimization problem.

\section{Multi-temporal decomposition model}

We consider the decomposition of a time series of SAR images into 3 components: a spatio-temporal background, additional point-like scatterers, and speckle noise. A multitemporal stack, represented by a vector $\mathbf{v}$, is decomposed by jointly estimating the background $\mathbf{u}_{\mathrm{B}}$ and the additional scatterers $\mathbf{u}_{\mathbf{S}}$. The speckle component $\mathbf{n}$ then corresponds to the ratio between observed amplitudes and the estimated radiometries, according to the multiplicative speckle model:

$$
\mathbf{v}=\left(\mathbf{u}_{\mathbf{B}}+\mathbf{u}_{\mathbf{S}}\right) \times \mathbf{n},
$$

where the multiplication is applied component-wise.

To apply the joint estimation of the background and detection of scatterers described in the preceding sections, the regularization term $\psi\left(\mathbf{u}_{\mathbf{B}}\right)$ needs to be set. Apart from the presence of many strong-scatterers, SAR images can reasonably well be described by smooth regions (with few variations of the radiometry) separated by sharp edges. In a time series, changes with time are rather smooth (e.g., vegetation changes) and pixels within a region of homogeneous radiometry generally keep comparable radiometries. When an abrupt change occurs, it is important to prevent from smoothing in time its apparition. We consider using a spatio-temporal total variation to impose some spatial and temporal smoothing while preserving sharp 
edges / changes. The proposed spatio-temporal regularization is:

$$
\begin{aligned}
\mathrm{TV}_{3 \mathrm{D}}^{\alpha}\left(\mathbf{u}_{\mathbf{B}}\right) & =\sum_{t} \sum_{i \sim j} w_{i, j}\left|u_{B t, i}-u_{B t, j}\right| \\
& +\alpha \sum_{i} \sum_{t}\left|u_{B t+1, i}-u_{B t, i}\right|,
\end{aligned}
$$

where $\alpha$ is the weight along the temporal direction.

If $\alpha$ is set to a very large value, no temporal change is possible and a constant (in time) background component is estimated. We denote $\mathrm{TV}_{3 \mathrm{D}}^{\infty}$ the regularization in this simplified case of a time-invariant background.

With Rayleigh likelihood and the $\mathrm{TV}_{3 \mathrm{D}}$ regularization, the joint decomposition amounts to solving the following minimization problem:

$$
\begin{array}{r}
\underset{\mathbf{u}_{\mathbf{S}} \geq 0, \mathbf{u}_{\mathbf{B}} \geq 0}{\arg \min } \sum_{i, t}\left[2 \log \left(u_{B t, i}+u_{S t, i}\right)+\frac{v_{t, i}^{2}}{\left(u_{B t, i}+u_{S t, i}\right)^{2}}\right] \\
+\lambda\left\|\mathbf{u}_{\mathbf{S}}\right\|_{0}+\beta \mathbf{T V}_{3 \mathrm{D}}^{\alpha}\left(\mathbf{u}_{\mathbf{B}}\right) .
\end{array}
$$

\section{OPTIMIZATION USING GRAPH-CUTS}

\section{A. Exact discrete optimization}

To apply the graph-cuts combinatorial optimization method to the minimization problem defined in equation (12), we need it to be formulated as:

$$
\underset{\mathbf{x}}{\arg \min } \sum_{t, i} f_{0}\left(x_{i, t}\right)+\sum_{(i, t) \sim\left(i^{\prime}, t^{\prime}\right)} f_{1}\left(x_{i, t}, x_{i^{\prime}, t^{\prime}}\right),
$$

i.e., as the sum of separable terms $f_{0}$ and pairwise terms $f_{1}$ involving (spatio-temporal) neighbors $(i, t)$ and $\left(i^{\prime}, t^{\prime}\right)$.

For any fixed background $\mathbf{u}_{\mathrm{B}}$, the equivalence between the estimation formulation (4) and the detection formulation (3) shows that the computation of the optimal vector of scatterers $\mathbf{u}_{\mathbf{S}}{ }^{\star}$ is readily obtained by simple testing against a threshold, for each pixel. It follows from (7) that the optimal $\mathbf{u}_{\mathbf{S}}$ is given by (see Appendix):

$$
u_{S_{t, i}^{\star}}\left(u_{B t, i}\right)= \begin{cases}v_{t, i}-u_{B t, i} & \text { if } v_{t, i}>u_{B t, i} \\ & \text { and } \frac{v_{i}^{2}}{u_{B}^{2}}-\log \frac{v_{i}^{2}}{u_{B}^{2}} \geq \lambda+1, \\ 0 & \text { otherwise. }\end{cases}
$$

By substituting $\mathbf{u}_{\mathbf{S}}$ with its optimal value $\mathbf{u}_{\mathbf{S}}{ }^{\star}\left(\mathbf{u}_{\mathbf{B}}\right)$ in the minimization problem (12), we get the sought formulation of equation (13), with

$$
\begin{aligned}
\mathbf{x} & =\mathbf{u}_{\mathbf{B}} \\
f_{0}\left(x_{i, t}\right) & =2 \log \left(u_{B t, i}+u_{S_{t, i}^{\star}}\left(u_{B t, i}\right)\right) \\
& +\frac{v_{t, i}^{2}}{\left(u_{B t, i}+u_{S_{t, i}^{\star}}\left(u_{B t, i}\right)\right)^{2}} \\
& +\lambda \cdot\left(u_{S_{t, i}^{\star}}^{\star}\left(u_{B t, i}\right) \neq 0\right) \\
f_{1}\left(x_{i, t}, x_{j, t}\right) & =\beta \cdot w_{i, j} \cdot\left|u_{B t, i}-u_{B t, j}\right| \\
f_{1}\left(x_{i, t}, x_{i, t+1}\right) & =\beta \cdot \alpha \cdot\left|u_{B t+1, i}-u_{B t, i}\right| .
\end{aligned}
$$

We apply the graph construction method of Ishikawa [42] to restate the minimization problem as the search of a cut in a graph. Finding the minimum cut in the graph using a maxflow algorithm will result in finding the decomposition minimizing the energy. We choose a (not necessarily uniform) quantization of values of the background: $\left\{q_{1}, q_{2}, \ldots, q_{\max }\right\}$. We then create a set of nodes representing all quantization levels for each (spatio-temporal) pixel, see Fig. 1(a). Oriented edges are created from the source to the nodes associated with level $q_{\max }$, then within a set of nodes associated to a given pixel, each node is connected to its preceding quantization level. Finally, the nodes representing level $q_{1}$ are connected to the sink. The capacity of the edge pointing to the node associated with pixel $(i, t)$ and quantization level $q_{c}$ is set to $f_{0}\left(q_{c}\right)$. Upstream edges with infinite capacity are also created to enforce that a minimum cut separates the source and the sink at a unique level for each pixel.

Spatial neighbors are then connected with bi-directional edges: two nodes representing the same quantization level $q_{c}$ are connected and the capacity of the edge is set to $\beta \cdot w_{i, j} \cdot\left(q_{c+1}-q_{c}\right)$. Finally, temporal neighbors are connected by creating bi-directional edges between nodes representing the same quantization level $q_{c}$, with a capacity set to $\alpha \cdot \beta$.

In figure 2 , we show the first and the last images obtained by applying the proposed decomposition on a SAR time series from Saint-Gervais (France) acquired by TerraSAR-X satellite.

1) Computational and memory complexity: The graph construction requires a large amount of memory that limits its application to small regions of interest and/or short time series. The number of vertices is proportional to the number of images in the time series, the number of pixels of each image, and the number of quantization levels. The number of edges is proportional to the number of nodes (about 8 times the number of nodes). We used the graph-cuts implementation described in [46]. In this graph implementation, each vertex requires 48 bytes of storage, and each edge requires 32 bytes. Hence, the memory footprint of the graph representation limits the size of the series of images that can be processed. For example, a series of 20 images of size $300 \times 400$ with a quantification into 50 levels requires $33.7 \mathrm{GiB}$ of RAM for the graph construction. However, when $\psi=\mathrm{TV}_{3 \mathrm{D}}^{\infty}$, the problem only involves one background. Solving the same problem with this regularization only requires $1.35 \mathrm{GiB}$, simplifying the exact optimization of the proposed model.

Regarding the computational cost, the worst-case complexity of the minimum cut is $O\left(E V^{2}|C|\right)$ where $C$ is the value of the minimum cut, $E$ is the number of edges and $V$ the number of vertices. In practice, the experimental complexity scales almost linearly with the number of nodes [46]. On a computer with an Intel $\AA$ Xeon(R) CPU E5-1620 with 16Gb of RAM, the algorithm takes $52,04 \mathrm{~s}$ to compute a decomposition on 2 images of $300 \times 400$ pixels with 50 levels of quantification. Note that we do not fully benefit from the power of the processor as the implementation of the algorithm used is single-core. Algorithms computing the grid-cut in parallel have also been proposed (see [47]) and more compact memory representations that exploit the regularity of the graph have been introduced in [48].

To reduce the computational and memory complexity, a 
(a)

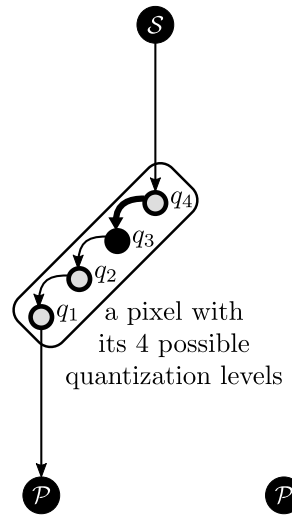

(b)

connections to the spatial neighbors

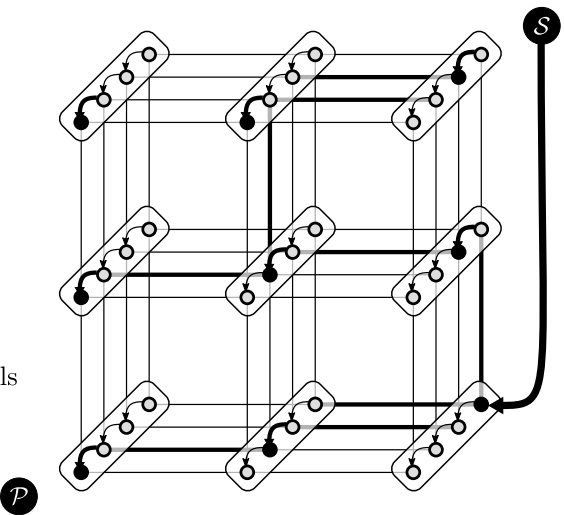

(c)

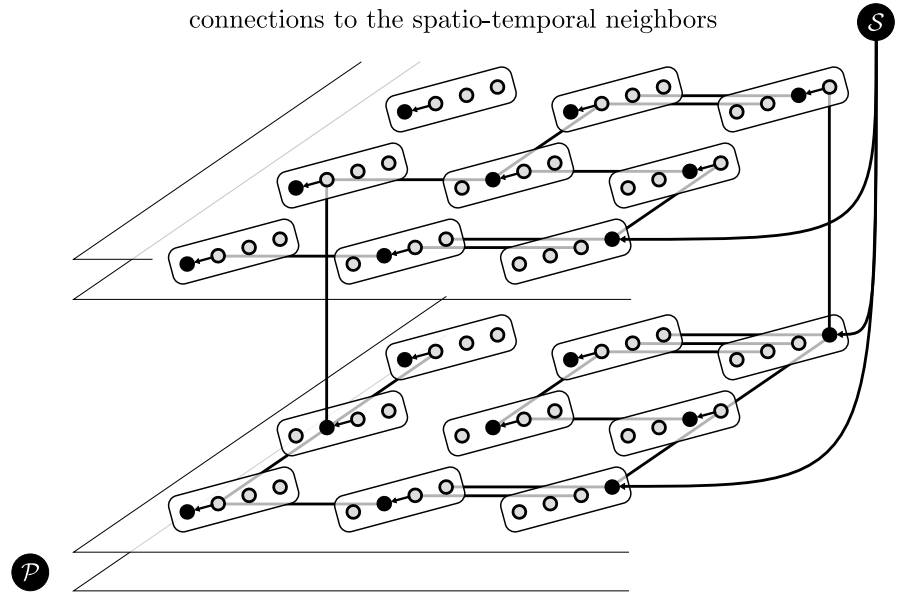

Fig. 1. Graph construction used to solve the image decomposition minimization problem. (a) A set of $q_{\max }$ nodes (one for each quantization level) is created for each pixel of the multi-temporal image $\mathbf{u}_{\mathbf{B}}$. Edges directed downstream are created, from the source $\mathcal{S}$ to the sink $\mathcal{P}$. The edge in the minimum cut, shown with a thick line, indicates that the quantization level right below (colored in black) is the optimal value. (b) Bi-directional edges are added between corresponding quantization levels for all pairs of spatially adjacent pixels (edges from the source and to the sink that are not in the minimum cut are omitted in the drawing). (c) Bi-directional edges are also added between temporal neighbors. Only edges in the minimum cut are shown here, with the corresponding optimal level at each pixel denoted by a black disk.

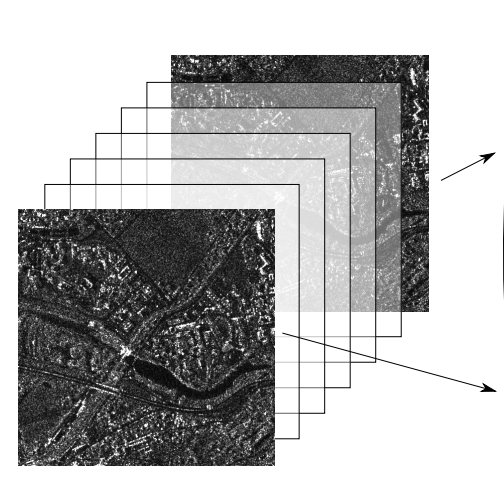

Multi-temporal SAR image (v)

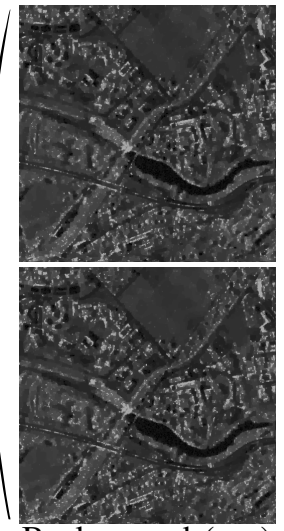

Background $\left(\mathbf{u}_{\mathbf{B}}\right)$

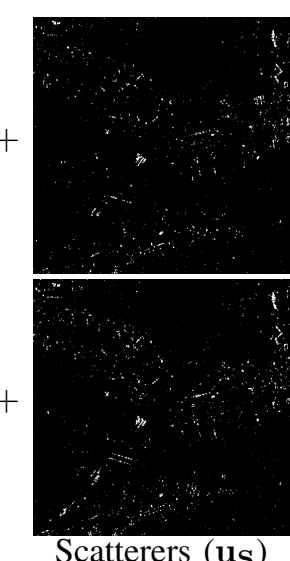

Scatterers $\left(\mathbf{u}_{\mathbf{S}}\right)$
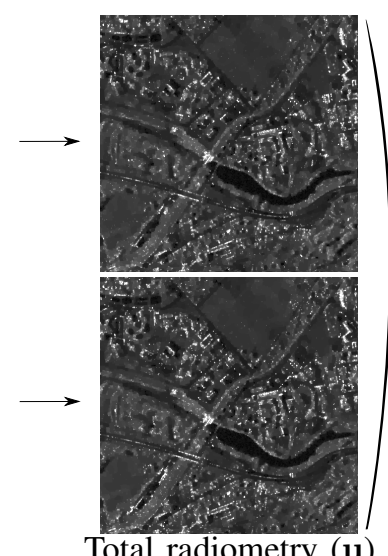

Total radiometry $(\mathbf{u})$

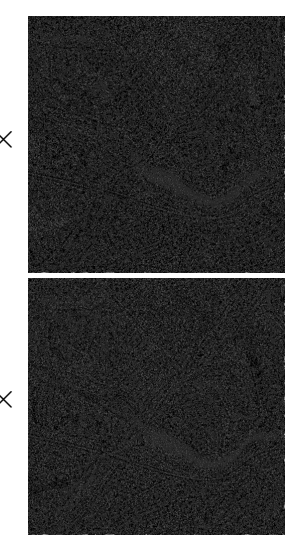

Speckle (n)

Fig. 2. Decomposition of a time series of TerraSAR-X images of Saint-Gervais, France. Only the first and the last image of the decomposition are shown.

subset of all quantization levels can be considered at a time to get an approximate solution, see [49], [50]. We describe in section III-B another approach based on block processing to further reduce memory requirements.

2) Quantization of the radiometry: The selection of the quantization levels used to represent the background radiometries $\mathbf{u}_{\mathrm{B}}$ requires to find a trade-off between accuracy (the more quantization levels the better) and the memory complexity (the graph footprint is proportional to the number of quantization levels). We used an adaptive method to set the quantization levels. We manually select the proportion $p$ of the image considered to be in the background (a typical value is around 95\%, but may be adapted according to the density of scatterers). The quantization levels are then computed from the quantiles of the $p$ lowest observed values of the first image. Note that only the background values $\mathbf{u}_{\mathrm{B}}$ need to be quantified, the bright scatterers component $\mathbf{u}_{\mathbf{S}}$ with the highest dynamic range is not quantified (see equation 14).

\section{B. Memory-efficient approximate optimization}

To apply the method to large images and/or long time series, it is necessary to develop a method for limited-memory graphcuts optimization.

Memory usage of the graph-cut method is proportional to the number of pixels in the series. The required memory can thus be reduced by computing the optimization locally, on spatio-temporal blocks extracted from the time series. Even though our model involves only second order cliques, limiting the direct interactions to the immediate neighborhood, the maximum a posteriori estimate involves long-range correlations, i.e., during the optimization, regularization effects are propagated over long distances. Simple division of the image into smaller blocks thus results into visible block artifacts, as can be observed in figure 3a. These block artifacts are due to the lack of context: a constant area in the global optimum that gets divided into two regions during the block-processing is represented by two different (constant) values with an artificial 


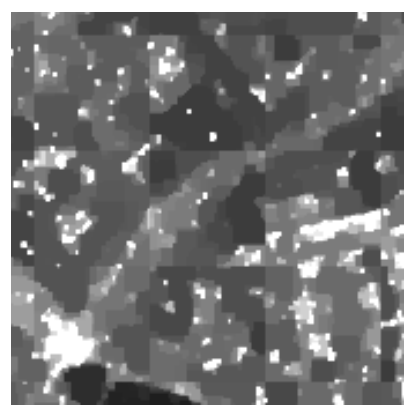

(a) Solution obtained by local optimization (computation window of size $50^{2}$ leading to a block effect)

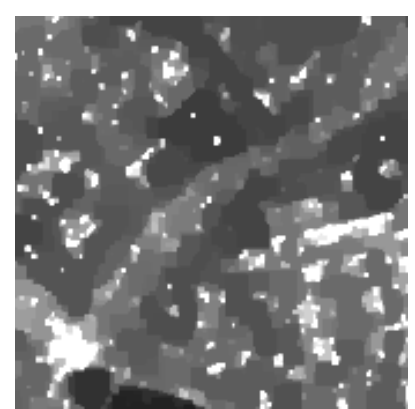

(b) Exact solution obtained using the presented algorithm for a computation window of size $150^{2}$

Fig. 3. Crops of the background component of the first image of the SaintGervais set obtained with the presented method (filling window of size $50^{2}$ ).

discontinuity between the blocks.

To introduce context in the window $F$ of interest, it is necessary to perform the optimization on a bigger window $C$ containing all the objects partially presents in $F$. The proposed method works as follows:

1) To process a given window $F$ (named filling window in the following), extract a larger computation window $C$ such that $F \subset C$.

2) Perform the optimization using the graph-cuts method described in section III-A on the spatio-temporal window $C$.

3) Keep the results of the decomposition only in the (smaller) filling window $F$.

4) Slide the filling window $F$ and repeat from step 1 until all the image has been covered by the filling window.

We show in figure 4 the root mean squared error obtained by the block-processing approach for different sizes of the computation window (the size of the block $F$ is kept constant and equal to $50 \times 50$ pixels for this single-date image). The required memory grows quadratically with the spatial window size. Note that as we increase the size of the computation window, the algorithm is slower. This explained by the fact that the number of cuts stay the same for any size of the computation window (as we do not change the size of $F$ ) while the size of the graph increases (which also increases the complexity of finding the minimal cut, see section III-A). As a comparison, running the optimal algorithm on the same data-set takes 505s. It can be observed that on this image, the exact solution is obtained for computation windows $C$ larger or equal to $150 \times 150$ pixels (which requires only $8.6 \%$ of the amount of memory needed to process the whole image at once). When the computation window $F$ is strictly smaller than the image size, the solution computed on the block $F$ is not guaranteed to match the solution obtained from the whole image, i.e., it is only approximate. Therefore, this algorithm allows to obtain another trade-off between memory/time complexity and quality of the result..

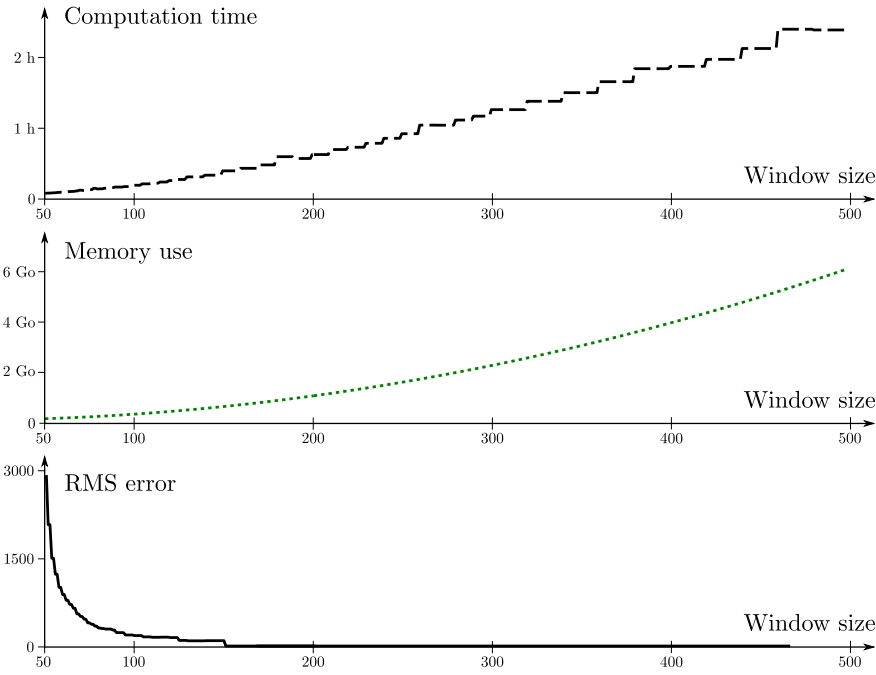

Fig. 4. Trade-off between computation time, memory use (top two graphs) and accuracy (bottom graph): evolution as a function of the size of the spatial computation window $C$, for the first image of the Saint-Gervais data-set.

\section{Advantage of the L0 PSEUdo-NORM OVER THE L1 RELAXATION}

The combinatorial optimization method described in section III is applicable to the L0 pseudo-norm and to the L1 norm, and provides in both cases the global optimum. It is thus possible to compare the performance of the two formulations: the L0 penalty derived from the GLRT and the L1 norm widely used to obtain a convex relaxation of the minimization problem, see for example [51].

We either consider the original minimization problem as stated in equation (12), or a modified minimization problem where the L0 pseudo-norm is replaced by the L1 norm. When considering the modified minimization problem involving the L1 norm, the optimal $\mathbf{u}_{\mathbf{S}}$ is no longer given by a simple expression as in equation (14). Optimal values $u_{S_{t, i}^{\star}}$ are either zero, or if strictly positive, they must cancel the first partial derivative of the sum of the log-likelihood and the L1 norm, given below:

$$
\begin{aligned}
\frac{\partial}{\partial u_{S}}\left[2 \log \left(u_{S}+u_{B}\right)\right. & \left.+\frac{v^{2}}{\left(u_{S}+u_{B}\right)^{2}}+\lambda u_{S}\right]= \\
& -\frac{2 v^{2}}{\left(u_{S}+u_{B}\right)^{3}}+\frac{2}{u_{S}+u_{B}}+\lambda .
\end{aligned}
$$

The only positive and real-valued root, as obtained by finding the roots of a third degree polynomial using a computer algebra system, is:

$$
u_{S}^{\star}\left(u_{B}\right)=t^{1 / 3}+\frac{4}{9 \lambda^{2} t^{1 / 3}}-\frac{3 \lambda u_{B}+2}{3 \lambda},
$$

with

$$
t=\frac{v \sqrt{27 \lambda^{2} v^{2}-16}}{3^{3 / 2} \lambda^{2}}+\frac{27 \lambda^{2} v^{2}-8}{27 \lambda^{3}} .
$$

We consider a numerical experiment to compare the detection performance of L0 and L1 formulations, for various contrasts between point-like scatterers and a piece-wise constant background, see figure $5 \mathrm{c}$. The contrast between each 


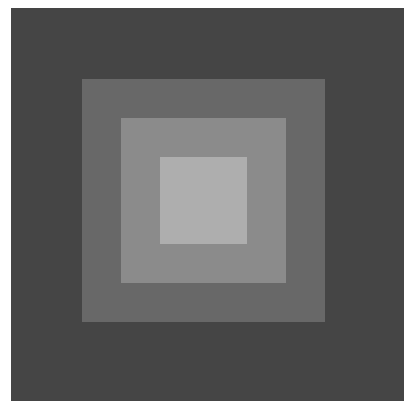

(a) Input noise-free background component

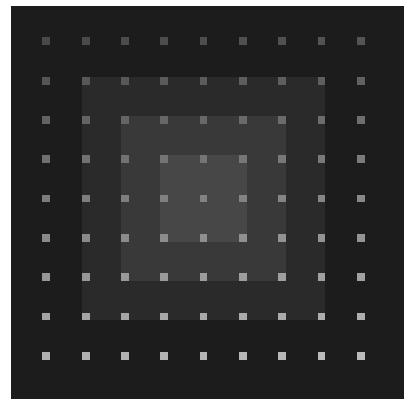

(c) Input noise-free image (figure 5a + figure $5 \mathrm{~b}$ )

Fig. 5. Input image used to compare LO and L1 models in a scatterer detection application in section IV. 100 noise realizations of figure $5 \mathrm{c}$ are generated and scores obtained in scatterers detection using both the LO and L1 models are presented in figure 6 .

scatterer and the background is given in figure 5d. 100 noisy versions are then generated using a Rayleigh multiplicative model. The L0 and L1 models are applied to each of these images with a fixed value of $\beta$ (set to 0.05 ) and various $\lambda$ values to obtain the receiver operating characteristic (ROC) curves drawn on figure 6 . They are compared with the ROC curve obtained using the method based on a local estimation of the background proposed in [27]. The L0 model outperforms both the L1 model and the detection based on local background estimation. We show in figure 7 the scatterers detected by each model, for an identical proportion of correct detections. The L0 model gives a uniform performance (correct detections and false detections are well distributed in the image), while the L1 model fails to detect scatterers in the regions with higher background radiometry and gives more false detections in low-radiometry areas. This phenomenon is confirmed by representing the evolution of the probability of false alarm (Pfa) and the probability of detection (Pd) as a function of the background radiometry, figure 8 . The L0 term produces constant Pfa and Pd (note that, in the numerical simulation, when the background radiometry changes, the scatterer radiometry is adapted so as to keep a unitary ratio: $u_{S} / u_{B}=1$ ). In contrast, the Pfa and Pd of the L1 model decrease when the background radiometry increases, which is consistent with the non-uniformity of detected scatterers observed in image $7 \mathrm{~b}$.

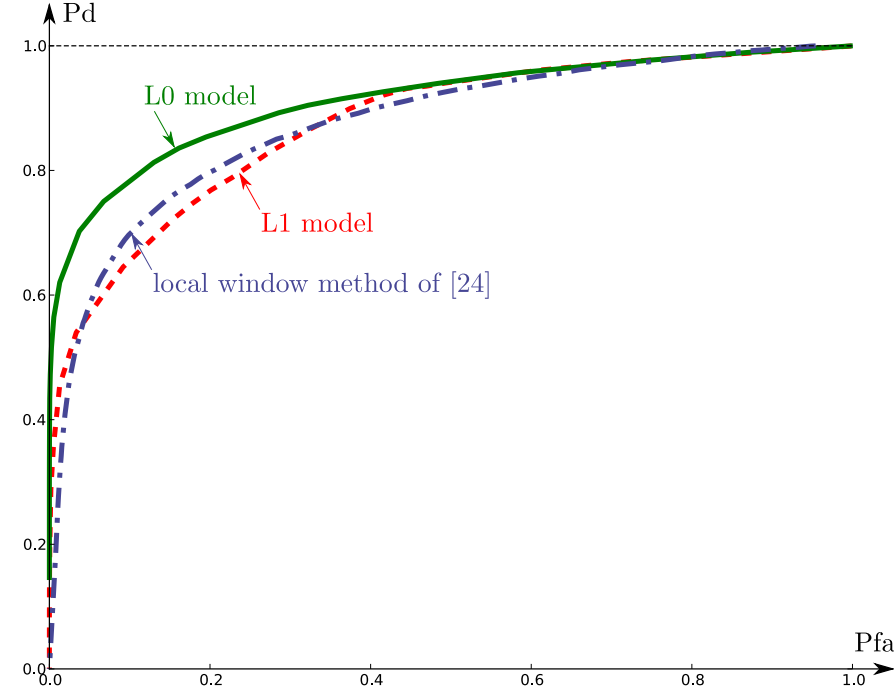

Fig. 6. Receiver operating curve (ROC) between L0 and L1 version of our model and a method based on local window analysis [27].

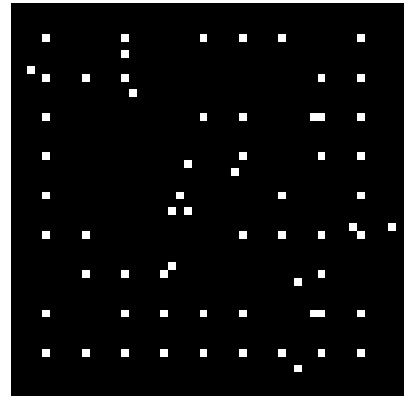

(a) Scatterers image obtained using the proposed decomposition (with LO term),$\lambda=2.5$

Fig. 7. 2 images of scatterers achieving the same probability of detection: left with an L0 penalty, right with an L1 penalty.

\section{APPLICATIONS OF THE DECOMPOSITION MODEL}

\section{A. Application to regularization}

In this section, we illustrate the interest of using our model to obtain a regularized version of the scene. The decomposition of a 56 images $\left(512^{2}\right.$ pixels each) stack of Paris acquired by TerraSar-X (spotlight mode, $1 \mathrm{~m} \times 1 \mathrm{~m}$ ) and co-registered using [52] is shown in figure 9.

We compare the results obtained by the two versions of our model (using either $\mathrm{TV}_{3 \mathrm{D}}^{1}$ or $\mathrm{TV}_{3 \mathrm{D}}^{\infty}$ as the prior on the

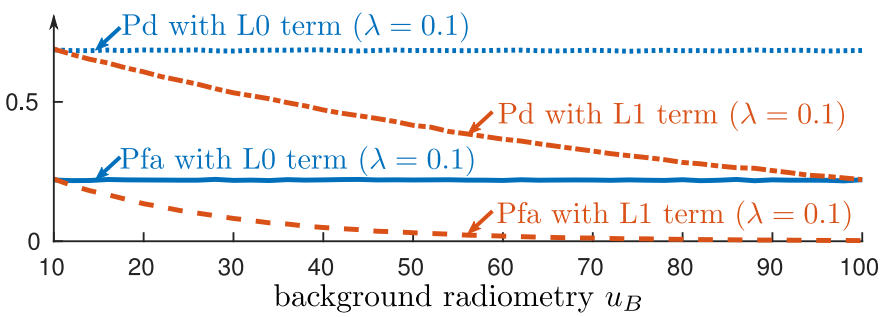

Fig. 8. With an L1 penalty, the probability of false alarm Pfa and the probability of detection Pd vary with the background radiometry. 


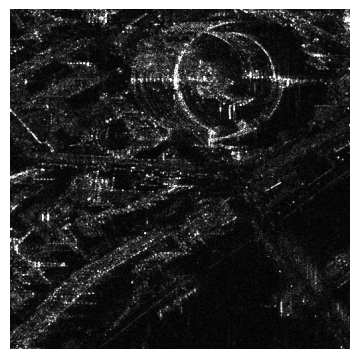

(a) Input image

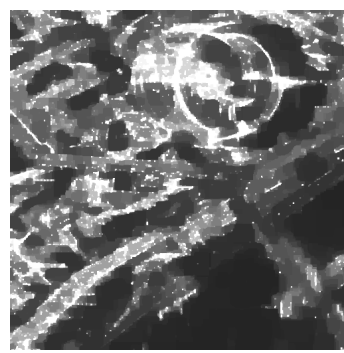

(f) Total variation (single date)

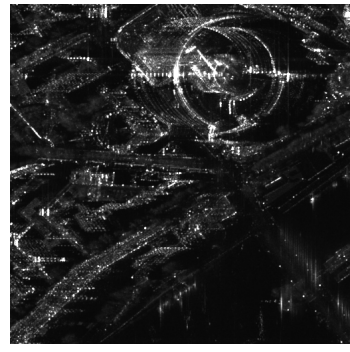

(k) 56 dates multi-looking (no decomposition)

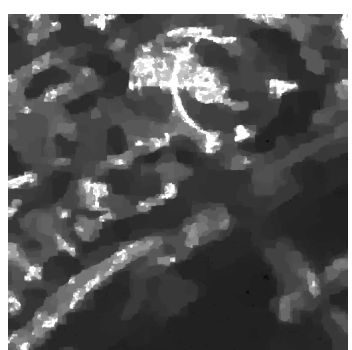

(b) Single date (Background)

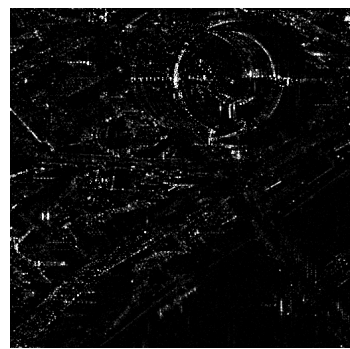

(g) Single date (Scatterers)

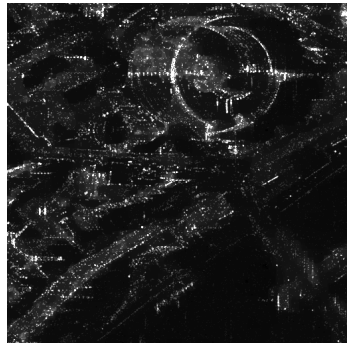

(1) Single date (background + scatterers)

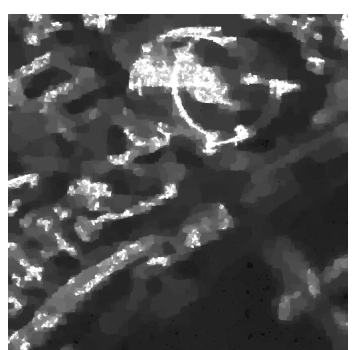

(c) 2 dates (Background, $\mathrm{TV}_{3 \mathrm{D}}^{1}$ )

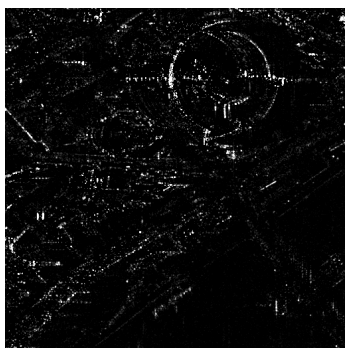

(h) 2 dates (Scatterers image, $\mathrm{TV}_{3 \mathrm{D}}^{1}$ )

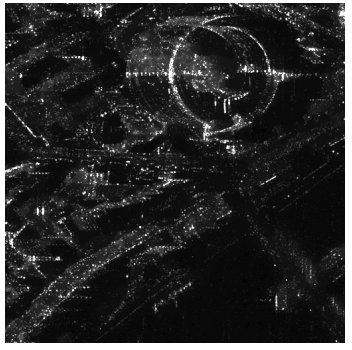

(m) 2 dates (Background + scatterers image, $\mathrm{TV}_{3 \mathrm{D}}^{1}$ )

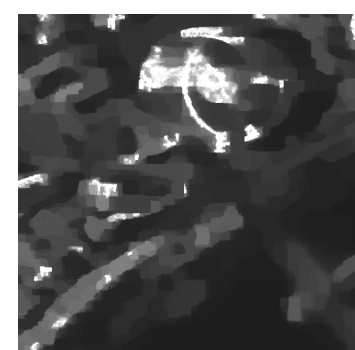

(d) 56 dates (Background, $\mathrm{TV}_{3 \mathrm{D}}^{1}$ )

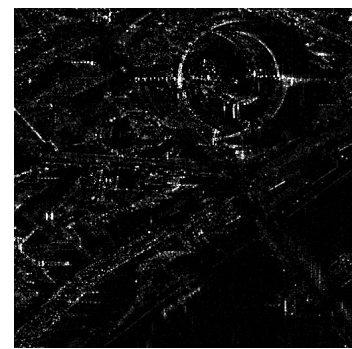

(i) 56 dates (Scatterers image, $\mathrm{TV}_{3 \mathrm{D}}^{1}$ )

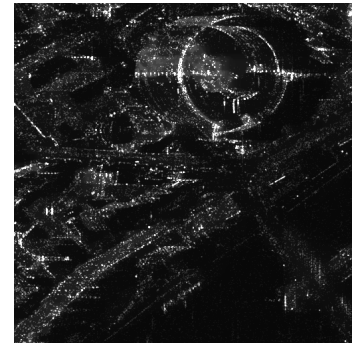

(n) 56 dates (Background + scatterers image, $\mathrm{TV}_{3 \mathrm{D}}^{1}$ )

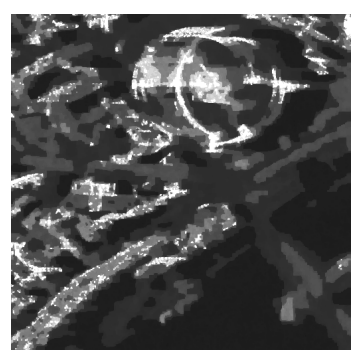

(e) 56 dates (Background, $\mathrm{TV}_{3 \mathrm{D}}^{\infty}$ )

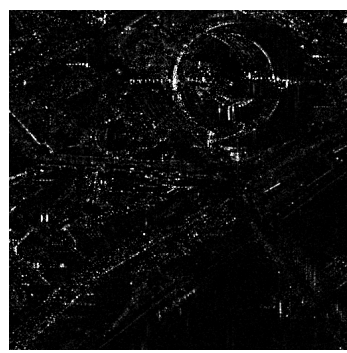

(j) 56 dates (Scatterers image, $\mathrm{TV}_{3 \mathrm{D}}^{\infty}$ )

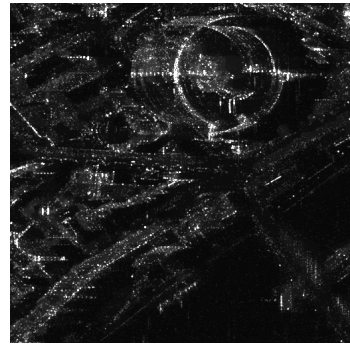

(o) 56 dates (Background + scatterers image, $\mathrm{TV}_{3 \mathrm{D}}^{\infty}$ )

Fig. 9. Comparison of the decomposition with different numbers of dates (1,2 or 56) with the classical TV regularization, temporal multi-looking. For the last 3 columns, results are given for the first image of the stack.

background, see section II-C) with different number of images $(1,2$ or 56$)$ and classical image regularization techniques: simple total variation regularization (without decomposition) and spatial multi looking. Note that when we consider only one image (second column), the model is the same as in [40]. As the regularization weight $\beta$ can not be set to the same value when using the different models, we have set it manually to obtain similar regularizations in the different results.

We can see that even though temporal multilooking (figure $9 \mathrm{k}$ ) gives the best results in terms of regularization, it suffers from punctual appearances of scatterers. This is mostly visible in the bottom-right of the image (which is the river "La Seine") where boats are present at some dates. Also note that this model loses the temporal information, at it results in only one image. Regarding total variation alone (figure 9f), we can see that we can not easily recognize objects in the background, as the strong scatterers tend to spread in their neighboring pixels.

Results from our model shows improvement compared to TV. Even when considering the single date version (figures 9b, $9 \mathrm{~g}$ and 9l, we can see that the background shows less scatterers, and that buildings are visible as an homogeneous area. Using a small stack (as in figures $9 \mathrm{c}, 9 \mathrm{~h}$ and $9 \mathrm{~m}$ ) shows only little improvement but allows for a more consistent regularization (same area from the two backgrounds will have similar values) than two separate runs on the single images. On the opposite, the improvement is visible when using large time series (such as in figures 9d, 9i and 9n). We can see that scatterers have almost disappeared from the background (allowing for a better distinction between the different areas) and the boundaries are more straight. The single background version of our model (figures $9 \mathrm{e}, 9 \mathrm{j}$ and 9o) shows a good regularization of the background, even though it is sometimes corrupted by strong scatterers.

Detailed results from this experience can be found at http://www.sylvainlobry.com/JSTARS-2015/.

\section{B. An application to change detection}

We propose a direct application of our decomposition model for change detection, under the hypothesis that changes mostly affect the scatterers. This assumption is likely to be fulfilled in urban or industrial areas. 
Given a time-series $\mathbf{v}$, the model described in section II-C provides multi-temporal decomposition $\left\{\left(\mathbf{u}_{\mathbf{B} 1}, \mathbf{u}_{\mathbf{S}_{1}}, \ldots\right.\right.$, $\left.\left.\mathbf{u}_{\mathbf{B} n}, \mathbf{u}_{\mathbf{S}_{n}}\right)\right\}$, or in the case of a time-invariant background $\left(\psi=\mathrm{TV}_{3 \mathrm{D}}^{\infty}\right.$ in equation 8$):\left\{\left(\mathbf{u}_{\mathbf{B}}, \mathbf{u}_{\mathbf{S}_{1}}\right), \ldots,\left(\mathbf{u}_{\mathbf{B}}, \mathbf{u}_{\mathbf{S}_{n}}\right)\right\}$. Change detection cannot be performed directly by pixel differences on the images of scatterers because the location and radiometry of scatterers fluctuate from one image to another even when insignificant changes happen in the scene. We therefore first binarize the scatterers images $\left\{\mathbf{u}_{\mathbf{S} t}\right\}$ (noted in the following $\left\{\mathbf{u}_{\mathbf{S}}{ }_{t}^{\text {bin }}\right\}$ ) to make the method insensitive to radiometric fluctuations. Robustness to small changes in the detected location of a scatterer is obtained by considering spatial neighborhoods:

$$
C_{t, t^{\prime}}(i)=\left|\sum_{\delta} \mathbf{u}_{\mathbf{s}_{t}}^{\mathrm{bin}}(i+\delta)-\sum_{\delta} \mathbf{u}_{\mathbf{s}_{t^{\prime}}}^{\text {bin }}(i+\delta)\right|
$$

where $\mathbf{u}_{\mathbf{S} t}$ and $\mathbf{u}_{\mathbf{S}^{\prime}}$ are the two images extracted from the decomposition of the time series, $\delta$ is a shift to iterate over the neighborhood of the pixel $i$ so $C_{t, t^{\prime}}(i)$ is the difference between the number of scatterers present in the window centered in $i$ in one image and the number of scatterers in the other one. This map is then thresholded to locate the changes between the two images. By constructing an histogram on $C_{t, t^{\prime}}$, the threshold can be chosen to reflect a user-defined percentage of changes.

To illustrate the results of our method, we ran this algorithm on a SAR time series from Saint-Gervais (France) acquired by TerraSAR-X satellite. This time series is composed of 26 single-look images of size $512^{2}$ (azimuth resolution: $2 m$, range resolution: $1 \mathrm{~m}$ ) coregistered using the method provided in [52] based on the fine knowledge of the sensors parameters provided by TerraSAR-X. 13 of these images have been sensed in 2009, 13 in 2011. The ground-truth used for the quantitative results have been labeled manually by [53]. We compare the results of this algorithm with other change detection methods in figure 10 and show an example of detected changes in figure 11 . The ROC curve is obtained by varying the threshold applied on $C_{t, t^{\prime}}$. Since all changes do not occur as scatterers changes, the ROC curves with our method do not reach high levels of probability of detection Pd. This simple thresholding approach achieves a performance comparable or superior to the methods described in [54] which apply a Maximum Likelihood (ML) test to segmented regions and [55] which applies a likelihood ratio locally at each pixel. Change detection based solely on the detected scatterers however is less accurate than the recent work by $\mathrm{Su}$ et al. [53] which use a likelihood ratio test after a non-local denoising step. Note that the simpler decomposition with a time-invariant background $\left(\psi=\mathrm{TV}_{3 \mathrm{D}}^{\infty}\right)$ gives worse detection results. A reason for this worse performance may come from the degradation of the detection of scatterers when the background is not correctly estimated, as is the case of regions where the background undergoes some changes.

\section{CONCLUSION}

This paper establishes the connection between a formulation of scatterers detection based on detection theory and

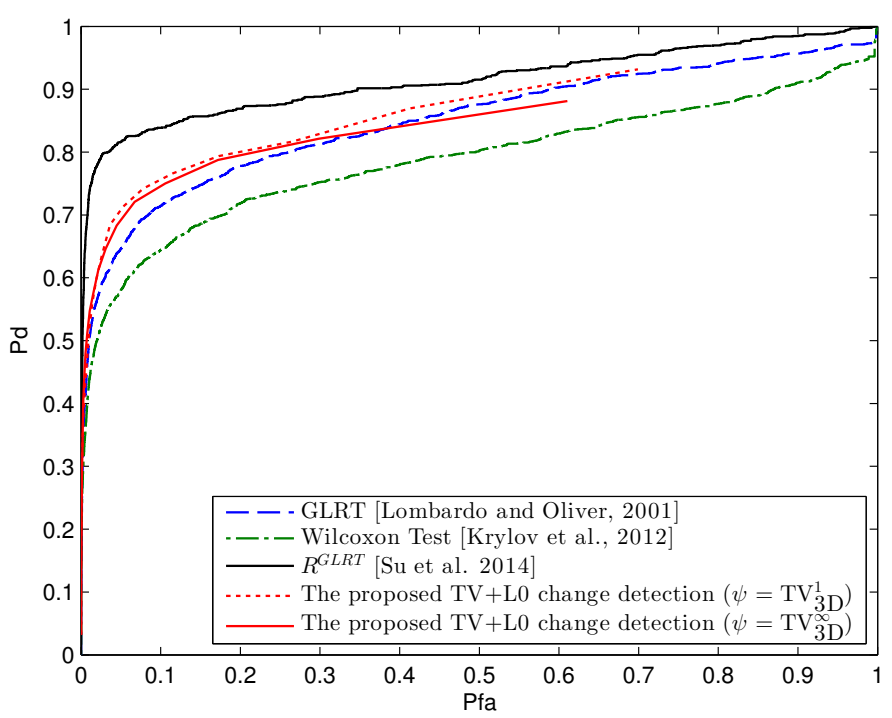

Fig. 10. ROC curve of various change detection algorithms

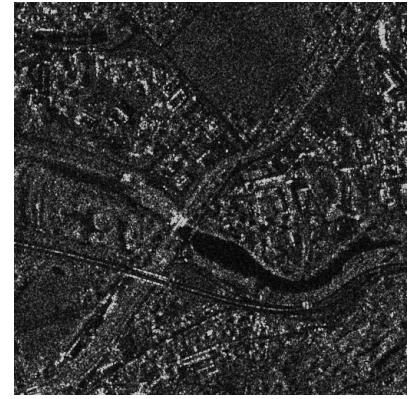

(a) Input image 1.

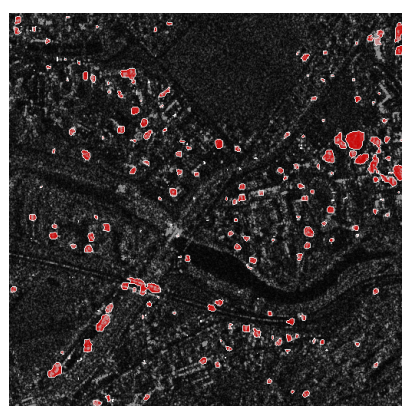

(c) Changes on image 1 .

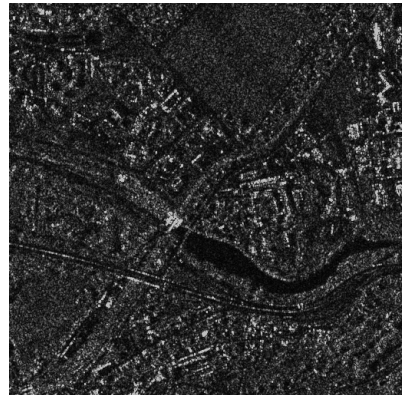

(b) Input image 26 .

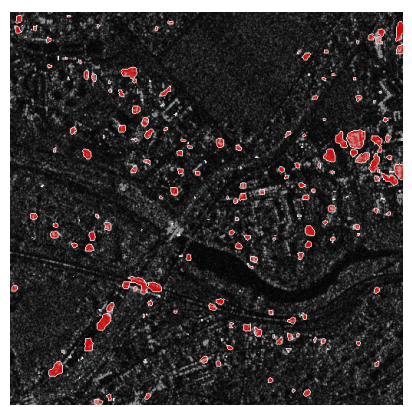

(d) Changes on image 26.
Fig. 11. Change detection results using the proposed method on images of Saint-Gervais series. Regions with changes that have been detected are circled in white.

the maximum a posteriori estimation with L0 pseudo-norm regularization. The problem of jointly detecting scatterers and estimating the background radiometry is then expressed as an image decomposition, stated as a combinatorial minimization problem. To solve this challenging large-scale combinatorial minimization problem, we propose to compute the minimumcut of a 4-D graph. Application to large areas and time series with numerous dates requires a limited memory algorithm based on block-processing with overlap. The comparison of the proposed L0-based decomposition with an L1-based 
decomposition shows a clear advantage for our formulation, which justifies tackling the combinatorial optimization problem. Our formulation gives constant false alarm rate and constant correct detection rate when the background level varies, in contrast to the L1 model. Finally, a simple application of the spatio-temporal decomposition is shown in the context of change detection.

Other decomposition models may be considered in the future, provided that they fulfill the constraints required to apply the graph-cut optimization method. Future work includes considering models designed for change detection rather than resorting to a post-processing step. The optimization step could be modified to use recent algorithms for min-cut computation (see [47], [48]). The fusion approach proposed in [56] could also be adapted to include information from an optical image in the computation of the multi-temporal background component.

\section{ACKNOWLEDGMENT}

The authors would like to thank the German Aerospace Agency (DLR) for the images (projects MTH0232, LAN 2708 and LAN1746). This work has been funded by the CNES and the "Futur et Ruptures" (Institut Mines-Télécom) program. L. Denis acknowledges a grant from CNRS (DETECTION DEFI IMAGIN).

\section{APPENDIX: DERIVATION OF RAYLEIGH GLRT}

To derive the expression of the GLRT under Rayleigh distribution, we begin by deriving the maximum likelihood estimator (MLE):

$$
\begin{aligned}
\hat{u}^{(\mathrm{MLE})} & =\underset{u}{\arg \max } \log \mathrm{p}(v \mid u) \\
& =\underset{u}{\arg \max } \log \left(\frac{2 v}{u^{2}}\right)-\frac{v^{2}}{u^{2}}
\end{aligned}
$$

The first-order optimality condition writes:

$$
\begin{aligned}
\frac{\partial}{\partial u} \log \mathrm{p}(v \mid u)=0 & \Leftrightarrow \frac{2 v^{2}}{u^{3}}-\frac{2}{u}=0 \\
& \Rightarrow u=v
\end{aligned}
$$

The MLE under Rayleigh distribution is simply $\hat{u}^{(\mathrm{MLE})}=v$.

We can now derive the GLRT:

Since $\max _{u_{S} \geq 0} \log \mathrm{p}\left(v_{i} \mid u_{B_{i}}+u_{S i}\right)=\log \left(\frac{2}{v_{i}}\right)-1$, the test $\max _{u_{S} \geq 0} \log \mathrm{p}\left(v_{i} \mid u_{B i}+u_{S i}\right) \underset{\mathcal{H}_{0}}{\stackrel{\mathcal{H}_{1}}{\gtrless}} \lambda+\log \left(\mathrm{p}\left(v_{i} \mid u_{B i}\right)\right)$ becomes:

$$
\frac{v_{i}^{2}}{u_{B i}^{2}}-\log \frac{v_{i}^{2}}{u_{B}^{2}} \underset{i}{\stackrel{\mathcal{H}_{1}}{\gtrless}} \lambda+1
$$

\section{REFERENCES}

[1] J. Lee, S. Cloude, K. Papathanassiou, M. Grunes, and I. Woodhouse, "Speckle filtering and coherence estimation of polarimetric SAR interferometry data for forest applications," IEEE Trans. Geosci. Remote Sens., vol. 41, no. 10, 2003.
[2] G. Vasile, E. Trouvé, and J. Lee, "Intensity-Driven AdaptiveNeighborhood Technique for Polarimetric and Interferometric SAR Parameters Estimation," IEEE Trans. Geosci. Remote Sens., vol. 44, no. $6,2006$.

[3] A. Buades, B. Coll, and J.-M. Morel, "A review of image denoising algorithms, with a new one," Multiscale Modeling \& Simulation, vol. 4, no. 2, pp. 490-530, 2005.

[4] C.-A. Deledalle, L. Denis, and F. Tupin, "Iterative weighted maximum likelihood denoising with probabilistic patch-based weights," IEEE Trans. Image Process., vol. 18, no. 12, pp. 2661-2672, 2009.

[5] — - "NL-InSAR: Nonlocal interferogram estimation," IEEE Trans. Geosci. Remote Sens., vol. 49, no. 4, pp. 1441-1452, 2011.

[6] J. Chen, Y. Chen, W. An, Y. Cui, and J. Yang, "Nonlocal filtering for polarimetric SAR data: A pretest approach," IEEE Trans. Geosci. Remote Sens., vol. 49, no. 5, 2011.

[7] H. Zhong, Y. Li, and L. Jiao, "SAR Image Despeckling Using Bayesian Nonlocal Means Filter With Sigma Preselection," IEEE Geosci. Remote Sens. Lett., vol. 8, no. 4, 2011.

[8] S. Parrilli, M. Poderico, C. Angelino, and L. Verdoliva, "A Nonlocal SAR Image Denoising Algorithm Based on LLMMSE Wavelet Shrinkage," IEEE Trans. Geosci. Remote Sens., vol. 50, no. 2, 2012.

[9] D. Cozzolino, S. Parrilli, G. Scarpa, G. Poggi, and L. Verdoliva, "Fast Adaptive Nonlocal SAR Despeckling," Geoscience and Remote Sensing Letters, IEEE, vol. 11, no. 2, pp. 524-528, Feb 2014.

[10] C.-A. Deledalle, L. Denis, F. Tupin, A. Reigber, and M. Jäger, "NL-SAR: A Unified Nonlocal Framework for Resolution-Preserving (Pol)(In)SAR Denoising," Geoscience and Remote Sensing, IEEE Transactions on, vol. 53, no. 4, pp. 2021-2038, April 2015.

[11] A. Achim, P. Tsakalides, and A. Bezerianos, "SAR image denoising via Bayesian wavelet shrinkage based on heavy-tailed modeling," IEEE Trans. Geosci. Remote Sens., vol. 41, no. 8, 2003.

[12] F. Argenti, T. Bianchi, and L. Alparone, "Multiresolution MAP Despeckling of SAR Images Based on Locally Adaptive Generalized Gaussian pdf Modeling," IEEE Trans. Image Process., vol. 15, no. 11, pp. 33853399, 2006.

[13] H. Xie, L. Pierce, and F. Ulaby, "SAR speckle reduction using wavelet denoising and Markov random field modeling," IEEE Trans. Geosci. Remote Sens., vol. 40, no. 10, pp. 2196-2212, Jan. 2002.

[14] L. I. Rudin, S. Osher, and E. Fatemi, "Nonlinear total variation based noise removal algorithms," Physica D: Nonlinear Phenomena, vol. 60, no. 1, pp. 259-268, 1992.

[15] J.-F. Aujol, G. Aubert, L. Blanc-Féraud, and A. Chambolle, "Image decomposition application to SAR images," in Scale Space Methods in Computer Vision. Springer, 2003, pp. 297-312.

[16] L. Denis, F. Tupin, J. Darbon, and M. Sigelle, "SAR image regularization with fast approximate discrete minimization," Image Processing, IEEE Transactions on, vol. 18, no. 7, pp. 1588-1600, 2009.

[17] F. Palsson, J. R. Sveinsson, M. Ulfarsson, and J. A. Benediktsson, "SAR image denoising using total variation based regularization with SUREbased optimization of the regularization parameter," in Geoscience and Remote Sensing Symposium (IGARSS), 2012 IEEE International, July 2012, pp. 2160-2163.

[18] G. Aubert and J.-F. Aujol, "A variational approach to removing multiplicative noise," SIAM Journal on Applied Mathematics, vol. 68, no. 4, pp. 925-946, 2008.

[19] G. Steidl and T. Teuber, "Removing multiplicative noise by douglasrachford splitting methods," Journal of Mathematical Imaging and Vision, vol. 36, no. 2, pp. 168-184, 2010.

[20] J. M. Bioucas-Dias and M. A. Figueiredo, "Multiplicative noise removal using variable splitting and constrained optimization," Image Processing, IEEE Transactions on, vol. 19, no. 7, pp. 1720-1730, 2010.

[21] F. Argenti, A. Lapini, T. Bianchi, and L. Alparone, "A tutorial on speckle reduction in synthetic aperture radar images," Geoscience and Remote Sensing Magazine, IEEE, vol. 1, no. 3, pp. 6-35, 2013.

[22] C.-A. Deledalle, L. Denis, G. Poggi, F. Tupin, and L. Verdoliva, "Exploiting Patch Similarity for SAR Image Processing: The nonlocal paradigm," Signal Processing Magazine, IEEE, vol. 31, no. 4, pp. 69-78, July 2014.

[23] R. Touzi, "A review of speckle filtering in the context of estimation theory," Geoscience and Remote Sensing, IEEE Transactions on, vol. 40, no. 11, pp. 2392-2404, 2002.

[24] G. Vasile, J.-P. Ovarlez, F. Pascal, and C. Tison, "Coherency matrix estimation of heterogeneous clutter in high-resolution polarimetric sar images," Geoscience and Remote Sensing, IEEE Transactions on, vol. 48, no. 4, pp. 1809-1826, 2010. 
[25] F. Pascal, Y. Chitour, J.-P. Ovarlez, P. Forster, and P. Larzabal, "Covariance structure maximum-likelihood estimates in compound gaussian noise: Existence and algorithm analysis," Signal Processing, IEEE Transactions on, vol. 56, no. 1, pp. 34-48, 2008.

[26] A. Lopes, E. Nezry, Goze, R. Touzi, and A. Solaas, "Adaptive processing of multilook complex SAR images," in Geoscience and Remote Sensing Symposium, 1992. IGARSS '92. International, vol. 2, May 1992, pp. 890-892.

[27] A. Lopes, E. Nezry, R. Touzi, and H. Laur, "Structure detection and statistical adaptive speckle filtering in SAR images," International Journal of Remote Sensing, vol. 14, no. 9, pp. 1735-1758, 1993.

[28] M. Cetin and W. C. Karl, "Feature-enhanced synthetic aperture radar image formation based on nonquadratic regularization," Image Processing, IEEE Transactions on, vol. 10, no. 4, pp. 623-631, 2001.

[29] J. H. Ender, "On compressive sensing applied to radar," Signal Processing, vol. 90, no. 5, pp. 1402-1414, 2010.

[30] L. C. Potter, E. Ertin, J. T. Parker, and M. Cetin, "Sparsity and compressed sensing in radar imaging," Proceedings of the IEEE, vol. 98, no. 6, pp. 1006-1020, 2010.

[31] X. X. Zhu and R. Bamler, "Tomographic sar inversion by-norm regularization - the compressive sensing approach," Geoscience and Remote Sensing, IEEE Transactions on, vol. 48, no. 10, pp. 3839-3846, 2010.

[32] K. R. Varshney, M. Cetin, J. W. Fisher III, and A. S. Willsky, "Sparse representation in structured dictionaries with application to synthetic aperture radar," Signal Processing, IEEE Transactions on, vol. 56, no. 8 , pp. 3548-3561, 2008.

[33] A. Ferretti, A. Fumagalli, F. Novali, C. Prati, F. Rocca, and A. Rucci, "A new algorithm for processing interferometric data-stacks: Squeesar," Geoscience and Remote Sensing, IEEE Transactions on, vol. 49, no. 9, pp. 3460-3470, 2011.

[34] G. Fornaro, D. Reale, and F. Serafino, "Four-dimensional sar imaging for height estimation and monitoring of single and double scatterers," Geoscience and Remote Sensing, IEEE Transactions on, vol. 47, no. 1, pp. 224-237, 2009.

[35] Y. Yamaguchi, A. Sato, W.-M. Boerner, R. Sato, and H. Yamada, "Fourcomponent scattering power decomposition with rotation of coherency matrix," Geoscience and Remote Sensing, IEEE Transactions on, vol. 49 , no. 6, pp. 2251-2258, 2011.

[36] J. Aujol, G. Gilboa, T. Chan, and S. Osher, "Structure-texture image decomposition: modeling, algorithms, and parameter selection," IJCV, vol. 67, no. 1, 2006.

[37] W. Yin, D. Goldfarb, and S. Osher, "Image Cartoon-Texture Decomposition and Feature Selection Using the Total Variation Regularized $L^{1}$ Functional," LNCS, vol. 3752, p. 73, 2005.

[38] M. Elad, J. Starck, P. Querre, and D. Donoho, "Simultaneous cartoon and texture image inpainting using morphological component analysis (MCA)," App. Comp. Harm. Anal., vol. 19, no. 3, pp. 340-358, 2005.

[39] J. Gilles, "Image Decomposition: Theory, Numerical Schemes, and Performance Evaluation," Adv. in Imag. Electron Phys., vol. 158, pp. 89-137, 2009.

[40] L. Denis, F. Tupin, and X. Rondeau, "Exact discrete minimization for TV+L0 image decomposition models," in Image Processing (ICIP), 2010 17th IEEE International Conference on. IEEE, 2010, pp. 25252528.

[41] S. Lobry, L. Denis, and F. Tupin, "Sparse \& smooth decomposition models for multi-temporal SAR images," in Analysis of Multitemporal Remote Sensing Images (Multi-Temp), 2015 8th International Workshop on the, July 2015, pp. 1-4.

[42] H. Ishikawa, "Exact optimization for Markov random fields with convex priors," Pattern Analysis and Machine Intelligence, IEEE Transactions on, vol. 25 , no. 10 , pp. 1333-1336, 2003.

[43] S. Kay, "Fundamentals of statistical signal processing, Vol. II: Detection Theory," Prentice Hall, 1998.

[44] J. W. Goodman, Speckle phenomena in optics: theory and applications. Roberts and Company Publishers, 2007.

[45] Y. Boykov and V. Kolmogorov, "Computing geodesics and minimal surfaces via graph cuts," in Computer Vision, 2003. Proceedings. Ninth IEEE International Conference on. IEEE, 2003, pp. 26-33.

[46] _ - "An experimental comparison of min-cut/max-flow algorithms for energy minimization in vision," Pattern Analysis and Machine Intelligence, IEEE Transactions on, vol. 26, no. 9, pp. 1124-1137, 2004

[47] J. Liu and J. Sun, "Parallel graph-cuts by adaptive bottom-up merging," in Computer Vision and Pattern Recognition (CVPR), 2010 IEEE Conference on, June 2010, pp. 2181-2188.

[48] O. Jamriska, D. Sykora, and A. Hornung, "Cache-efficient graph cuts on structured grids," in Computer Vision and Pattern Recognition (CVPR), 2012 IEEE Conference on, June 2012, pp. 3673-3680.
[49] L. Denis, F. Tupin, J. Darbon, and M. Sigelle, "SAR image regularization with fast approximate discrete minimization," Image Processing, IEEE Transactions on, vol. 18, no. 7, pp. 1588-1600, July 2009.

[50] A. Shabou, J. Darbon, and F. Tupin, "A Markovian Approach for InSAR Phase Reconstruction With Mixed Discrete and Continuous Optimization," Geoscience and Remote Sensing Letters, IEEE, vol. 8, no. 3, pp. 527-531, May 2011.

[51] J. Tropp et al., "Just relax: Convex programming methods for identifying sparse signals in noise," Information Theory, IEEE Transactions on, vol. 52, no. 3, pp. 1030-1051, 2006.

[52] J.-M. Nicolas, E. Trouve, R. Fallourd, F. Vernier, F. Tupin, O. Harant, M. Gay, and L. Moreau, "A first comparison of Cosmo-Skymed and TerraSAR-X data over Chamonix Mont-Blanc test-site," in Geoscience and Remote Sensing Symposium (IGARSS), 2012 IEEE International. IEEE, 2012, pp. 5586-5589.

[53] X. Su, C.-A. Deledalle, F. Tupin, and H. Sun, "NORCAMA: Change Analysis in SAR Time Series by Likelihood Ratio Change Matrix Clustering," ISPRS Journal of Photogrammetry and Remote Sensing, pp. 247-261, May 2014.

[54] P. Lombardo and C. Oliver, "Maximum likelihood approach to the detection of changes between multitemporal SAR images," IEEE Proceedings-Radar, Sonar and Navigation, vol. 148, no. 4, pp. 200 210, 2001.

[55] V. Krylov, G. Moser, A. Voisin, B. Serpico, Sebastiano, and J. Zerubia, "Change detection with Synthetic Aperture Radar images by Wilcoxon statistic likelihood ratio test," in IEEE International Conference on Image Processing 2012, Orlando, United States, Sep 2012.

[56] L. Denis, F. Tupin, J. Darbon, and M. Sigelle, "Joint Regularization of Phase and Amplitude of InSAR Data: Application to 3-D Reconstruction," Geoscience and Remote Sensing, IEEE Transactions on, vol. 47, no. 11, pp. 3774-3785, Nov 2009. 